To summarize, the analytical approach described by Maei et al. (2009) provides a more effective and accurate new measure in terms of its superb sensitivity and statistical validity in water maze studies. Furthermore, the $\mathrm{H}$ measure will contribute greatly to the advancement of behavioral assays that, in conjunction with molecular genetic approaches, are designed to elucidate the molecular mechanisms underlying learning and memory.

\section{REFERENCES}

D'Hooge, R., and De Deyn, P. P. (2001). Applications of the Morris water maze in the study of learning and memory. Brain Res. Brain Res. Rev. 36, 60-90.
Gallagher, M., and Rapp, P. R. (1997). The use of animal models to study the effects of aging on cognition. Annu. Rev. Psychol. 48, 339-370.

Lipp, H. P., and Wolfer, D. P. (1998). Genetically modified mice and cognition. Curr. Opin. Neurobiol. 8, 272-280.

Maei, H. R., Zaslavsky, K., Teixeira, C. M., and Frankland, P. W. (2009). What is the most sensitive measure of water maze probe test performance? Front. Integr. Neurosci. 3, 4. doi: 10.3389/neuro.07.004.2009.

Morris, R.G.M.(1981).Spatial localization does not require the presence of local cues. Learn. Motiv. 12, 239-260.

Morris, R. G. M. (1984). Developments of a water-maze procedure for studying spatial learning in the rat. $J$. Neurosci. Methods 11, 47-60.

Tanda, K., Nishi, A., Matsuo, N., Nakanishi, K., Yamasaki, N., Sugimoto, T., Toyama, K., Takao, K., and Miyakawa, T. (2009). Abnormal social behavior, hyperactivity, impaired remote spatial memory, and increased D1-mediated dopaminergic signaling in neuronal nitric oxide synthase knockout mice. $M o l$. Brain 2, 19.

Tsien, J. Z., Huerta, P. T., and Tonegawa, S. (1996). The essential role of hippocampal CA1 NMDA receptordependent synaptic plasticity in spatial memory. Cell 87, 1327-1338.

Vorhees, C. V., and Williams, M. T. (2006). Morris water maze: procedures for assessing spatial and related forms of learning and memory. Nat. Protoc. 1, 848-858.

Received: 21 September 2009; published: 15 April 2010. Citation: Front. Neurosci. (2010) 4, 1: 1-2. doi: 10.3389/ neuro.01.002.2010

Copyright $\odot 2010$ Lee and Kaang. This is an open-access publication subject to an exclusive license agreement between the authors and the Frontiers Research Foundation, which permits unrestricted use, distribution, and reproduction in any medium, provided the original authors and source are credited.

\title{
Decisions of voluntary action: what vs when
}

\section{Deborah J. Serrien* \\ School of Psychology, University of Nottingham, University Park, Nottingham, UK \\ *Correspondence: deborah.serrien@nottingham.ac.uk}

\section{A commentary on}

\section{Dissociating what and when of intentional actions}

by Veronika Krieghoff, Marcel Brass, Wolfgang Prinz and Florian Waszak

Voluntary action is a fundamental characteristic of human behavior and identifies our ability to realize intentionally driven tasks. Functional imaging work has indicated that intentional performance activates neural circuitry confined to the fronto-medial cortex (Cunnington et al., 2002; Lau et al., 2004; Nachev et al., 2005). This network activity integrates, besides the conscious experience of intending (Haggard and Clark, 2003; Lau et al., 2004), a series of processes in relation to the intentional act (Brass and Haggard, 2007). Accordingly, intentional behavior can be viewed as a form of decision making that consists of various components with different types of information processing (Brass and Haggard, 2008; Haggard, 2008). This distinctive viewpoint is important as voluntary action has often been regarded as a unitary concept without little con- sideration for the component functions. In this respect, it has been suggested that voluntary action has at least two decisional processes: what action to perform (selection component) and when to perform it (timing component), (Mueller et al., 2007). Thus, the "what" decision specifies which action to perform from a range of alternative options, whereas the "when" decision denotes the moment in time at which to execute the action.

In their recent contribution to Frontiers in Neuroscience, Krieghoff et al. (2009) presented an fMRI study that provides new insights into voluntary behavior. In particular, the authors proposed a welldesigned protocol in which they simultaneously and independently manipulated the selection and timing component within one experimental paradigm. Moreover, the participants were instructed to perform one of two possible actions at one of two possible moments in time. Furthermore, the action and moment in time were freely chosen or externally triggered by means of a cue. In other words, the "what" (selection) and "when" (timing) decision of the impending action were based on an internal judgment or imposed by an external stimulus. This distinction between internally and exter- nally generated responses is relevant as both types of activities are known to have (partly) dissociable neural circuitry in respect to the component process of selection, as well as timing (Deiber et al., 1999; Cunnington et al., 2002, 2006; Lau et al., 2006).

As the main objective of Krieghoff et al. (2009) was to investigate the decisional processes of voluntary behavior, the data analysis focused primarily on the cue-related activity that specified the decision making process rather than on the target-related activity that represented the implementation of that decision. In view of that premise, the whole-brain analysis of the cue-related signal showed that two fronto-medial wall areas associated with distinct component functions of voluntary action: whereas the selection component linked strongly with the rostral cingulate zone (RCZ), the timing component tied closely with the superior medial frontal gyrus (SFG), which is localized close to pre-SMA (Rushworth, 2008). These results indicate that separate brain regions are involved in distinct decisional processes, and accordingly highlight a functional dissociation of intentional action. However, an additional 
signal strength analysis revealed that both processes interact with one another. In particular, neural activity within the paramedial frontal cortex was increased for internal as compared to external timing, but only when action selection was specified externally. Combined, these observations challenge the idea of a unitary control system and underscore the existence of distinct, albeit interdependent, decisional processes that together shape voluntary action (Brass and Haggard, 2008). Of note is that this dependency is in accordance with the argument that aspects from the component processes have to be considered in order for an action and its consequences to be evaluated.

By introducing an innovative experimental paradigm, the work of Krieghoff et al. (2009) has provided a significant step into the understanding of intentional control by specifying dissociable brain regions that deal with component functions. Further research into the dynamics of voluntary action is necessary in order to detail the functional architecture of intentional behavior and its neural correlates.
Future studies may elaborate on the interregional influences and coordination of the brain areas that make up the network activity, and the means by which contextrelated factors modify intentional processing within the circuitry.

\section{REFERENCES}

Brass, M., and Haggard, P. (2007). To do or not to do: the neural signature of self-control. J. Neurosci. 27, 9141-9145.

Brass, M., and Haggard, P. (2008). The what, when, whether of intentional action. Neuroscientist 14 319-325.

Cunnington, R., Windischberger, C., Deecke, L., and Moser, E. (2002). The preparation and execution of self-initiated and externally-triggered movement: a study of event-related fMRI. Neuroimage 15 , 373-385.

Cunnington, R., Windischberger, C., Robinson, S., and Moser, E. (2006). The selection of intended actions and the observation of others' actions: a time-resolved fMRI study. Neuroimage 29, 1294-1302.

Deiber, M. P., Honda, M., Ibañez, V., Sadato, N., and Hallett, M. (1999). Mesial motor areas in self-initiated versus externally triggered movements examined with fMRI: effect of movement type and rate. J. Neurophysiol. 81, 3065-3077.

Haggard, P. (2008). Human volition: towards a neuroscience of will. Nat. Rev. Neurosci. 9, 934-946.
Haggard, P., and Clark, S. (2003). Intentional action: conscious experience and neural prediction. Conscious Cogn. 12, 695-707.

Krieghoff, V., Brass, M., Prinz, W., and Waszak, F. (2009). Dissociating what and when of intentional actions. Front. Hum. Neurosci. 3, 1-10. doi: 10.3389/ neuro.09.003.2009.

Lau, H., Rogers, R. D., and Passingham, R. E. (2006). Dissociating response selection and conflict in the medial frontal surface. Neuroimage 29, 446-451.

Lau, H. C., Rogers, R. D., Ramnani, N., and Passingham, R. E. (2004). Willed action and attention to the selection of action. Neuroimage 21, 1407-1415.

Mueller, V.A., Brass, M., Waszak, F., and Prinz, W. (2007). The role of the preSMA and the rostral cingulate zone in internally selected actions. Neuroimage 37, 1354-1361.

Nachev, P., Rees, G., Parton, A., Kennard, C., and Husain, M. (2005). Volition and conflict in human medial frontal cortex. Curr. Biol. 15, 122-128.

Rushworth, M. F. (2008). Intention, choice, and the medial frontal cortex. Ann. N. Y. Acad. Sci. 1124 181-207.

Received: 31 August 2009; published: 15 April 2010. Citation: Front. Neurosci. (2010) 4, 1: 2-3. doi: 10.3389/ neuro.01.001.2010

Copyright $($ C 2010 Serrien. This is an open-access publication subject to an exclusive license agreement between the authors and the Frontiers Research Foundation, which permits unrestricted use, distribution, and reproduction in any medium, provided the original authors and source are credited.

\title{
Forget before you remember: dynamic mechanism of memory decay and retrieval
}

\author{
Tatiana Engel* and David Andrieux ** \\ Department of Neurobiology and Kavli Institute for Neuroscience, Yale University School of Medicine, New Haven, CT, USA \\ *Correspondence: *tatiana.engel@yale.edu; **david.andrieux@yale.edu
}

\section{A commentary on}

Neurophysiological bases of exponential sensory decay and top-down memory retrieval: a model

by Ariel Zylberberg, Stanislas Dehaene, Gabriel B. Mindlin and Mariano Sigman

Sensory systems are confronted with a continuous stream of inputs, but only a small fraction of these sensory stimuli reaches our awareness, is consciously perceived and can be remembered. Perception is never driven solely by the bottom-up stimulation, but crucially depends on the top-down modulations. Top-down signals convey behavioral context, such as attention, expectation and perceptual task, and are reflected in the context-specific response modulation in single neurons (Miller and Cohen, 2001; Corbetta and Shulman, 2002). Top-down interactions can be of many different kinds: augmenting or multiplying responses, sharpening tuning curves, controlling contextual influences, or acting as a modulator of plasticity (Desimone and Duncan, 1995; Maunsell and Treue, 2006). Although a lot of empirical knowledge has been accumulated on how top-down interactions modulate neural responses, only a few theoretical attempts have been made so far to explain the underlying biophysical mechanisms and to bridge the gap between the behavioral and single-cell data (Buia and Tiesinga, 2006; Deco and Rolls, 2006; Ardid et al., 2007).

The recent study by Zylberberg et al. (2009) published in Frontiers in Computational Neuroscience aims to uncover these biophysical mechanisms in a particular setting of top-down memory retrieval. The authors try to answer several general and long-standing questions: How do the bottom-up and top-down signals interact to produce a perception? What are the neural mechanisms of effortless (iconic) vs voluntary (working) memory? 